\title{
Towards an ab initio calculation of elemental thermocouple output
}

\author{
Jonathan V. Pearce* \\ National Physical Laboratory, Hampton Road, Teddington, TW11 0LW, UK
}

Received: 9 March 2016 / Accepted: 10 April 2016

\begin{abstract}
A simple model is presented for the calculation of the temperature dependence of the thermopower of metals on an ab initio basis, which includes an accurate calculation of the electronic density of states. The aim is to predict the temperature dependence of the electromotive force generated by the elemental thermocouples $\mathrm{Au} / \mathrm{Pt}$ and $\mathrm{Pt} / \mathrm{Pd}$, by calculating the thermopower of $\mathrm{Pt}, \mathrm{Au}$, and $\mathrm{Pd}$. The model is compared with the measured thermopower in each case, and found to be qualitatively accurate. It is suggested that the accuracy of the model is limited principally by the ab initio calculation of the electronic density of states of the model.
\end{abstract}

\section{Introduction}

Thermocouples are the most widely used type of temperature sensor in industry. They consist of two thermoelements (wires) connected at one end to form the measuring junction. Often, the open end is held at a known temperature (the reference temperature). Essentially, an electromotive force (emf) is generated across the two thermoelements in response to a temperature gradient along the pair of thermoelements. The linear rate of change of emf with temperature for a pair of wires is given by the combined Seebeck coefficient.

Although thermocouples are simple in construction, they exhibit some very rich physics $[1-3]$. It is of considerable interest to construct a model to predict the relationship between the temperature at the measuring junction and the emf generated, for a given pair of materials. Currently, thermocouples must be calibrated with respect to known temperatures, and the temperature is then related to the emf by an empirical model parameterised by the calibration. By using an accurate ab initio model of the temperature dependence of the thermopower, the thermocouple could, in principle, be used as a primary thermometer; however, even a modestly accurate model is instructive and can assist with the selection of new types and ultimately the effect of changing composition (due to e.g. high temperature exposure or ionising radiation) on the output.

Here an analytical model is employed to generate an expression for the thermopower of a particular wire. The analytical expression requires in turn a knowledge of the electron density of states as a function of energy, and the Fermi energy as a function of temperature. The density of states is evaluated numerically using the

^ Correspondence: jonathan.pearce@npl.co.uk plane-wave density functional theory (DFT) code ABINIT [4-6]. This enables a numerical evaluation of the Fermi energy by balancing the density of states with the known number of valence electrons in the metal. The thermopower of the elements Pt, Au and Pd is then calculated, and the output of the elemental thermocouples $\mathrm{Au} / \mathrm{Pt}$ and $\mathrm{Pt} / \mathrm{Pd}$ is then calculated as a function of temperature.

\section{Theoretical background}

Here we describe a way, based on well established methods [2], to derive an expression for the temperature dependence of the Seebeck coefficient of a metal. If we apply an electric field $\varepsilon_{x}$ in the $x$-direction to a single crystal of metal, the current density in the positive $x$-direction per unit volume of material is

$$
j_{x}=\sum n e v_{x}
$$

where $n$ is the electron number density, $v$ is the velocity of the electron (with charge $e$ ) $v_{x}$ is its $x$-component and the summation is over all occupied states.

This can be written [2] in terms of $v$ and the relaxation time $\tau$ as:

$$
j_{x}=\frac{-e^{2} \varepsilon_{x}}{4 \pi^{3} \hbar} \iint \tau \frac{v_{x}^{2}}{v} d S \frac{d P}{d E} d E
$$

where the first integral is over a surface $S$ of constant energy $E$ and the second integral is over all energies. $P$ is the Fermi function describing the probability of occupation of a given state with energy $E$, at a temperature $T$, given the Fermi energy $E_{F}$ :

$$
P=\frac{1}{\exp \left[\left(E-E_{F}\right) / k_{B} T\right]+1}
$$


where $k_{B}$ is the Boltzmann constant. It is now convenient to introduce the partial electrical conductivity $\sigma$ :

$$
\sigma_{x}(E)=\frac{e^{2}}{4 \pi^{3} \hbar} \int \tau(E) v_{x}^{2}(e) \frac{d S}{v}
$$

where the integration is over a constant energy surface in $k$-space with energy $E$. $\sigma$ is in effect the conductivity of the conduction electrons with energy $E$. Then $j_{x}$ can be written:

$$
j_{x}=-\varepsilon_{x} \int \sigma_{x}(E) \frac{d P}{d E} d E .
$$

For the purpose of the current application, $\sigma_{x}$ can be simplified to:

$$
\sigma_{x}(E)=\frac{e^{2}}{4 \pi^{3} \hbar} \tau(E) \bar{v}_{x}^{2}(E) N(E)
$$

where $N(E)$ is the electron density of states. Note that in a cubic metal, $s_{x}$ and $v_{x}$ are isotropic so we omit the suffix $x$.

The electrons have different energies $E$ but a common free energy $E_{F}$, the Fermi energy, which characterises the entire distribution. Assuming that these properties of the electron gas at equilibrium are essentially unchanged even when the electrons flow to produce an electric current, then the thermal energy associated with the electron is related to the difference between the internal energy $E$ and the free energy $E_{F}$, i.e. $E-E_{F}$, where $E>E_{F}$.

The expression (5) for $j$ indicates that the total conductivity $(j / \varepsilon)$ is the sum of all the partial conductivities suitably weighted. It is then possible to write down the ratio of thermal energy flux to charge flux:

$$
T \frac{d V}{d T}=\frac{1}{e} \frac{\int_{-\infty}^{\infty} \sigma(E)\left(E-E_{F}\right)(d P / d E) d E}{\int_{-\infty}^{\infty} \sigma(E)(d P / d E) d E} .
$$

Now, the derivative of the Fermi function, $d P / d E$, acts to select out the density of states over a very narrow range of energies centred at $E_{F}$. The quantities $\tau(E)$ and $v(E)$, and their energy dependence, are not practically calculable, so the assumption is made that they do not vary with energy over the range selected by the Fermi occupation function (3). Then most of the terms in (6) cancel, and the thermopower $d V / d T$ can be expressed in terms of the electron density of states (see also [7]):

$$
\frac{d V}{d T}=\frac{1}{e T} \frac{\int_{-\infty}^{\infty} N(E)\left(E-E_{F}\right)(d P / d E) d E}{\int_{-\infty}^{\infty} N(E)(d P / d E) d E}
$$

Note also that since $d P / d E$ is only significant for $E \approx E_{F}$ this result is approximately equal to:

$$
\frac{d V}{d T}=-\frac{1}{e} \frac{d E_{F}}{d T}
$$

which is a much simpler expression to evaluate. A comparison of the behaviour of (8) and (9) shows that the simplified method is a good approximation to the full method. In the following sections, an analysis using the full method (8) is presented to maximise the accuracy.
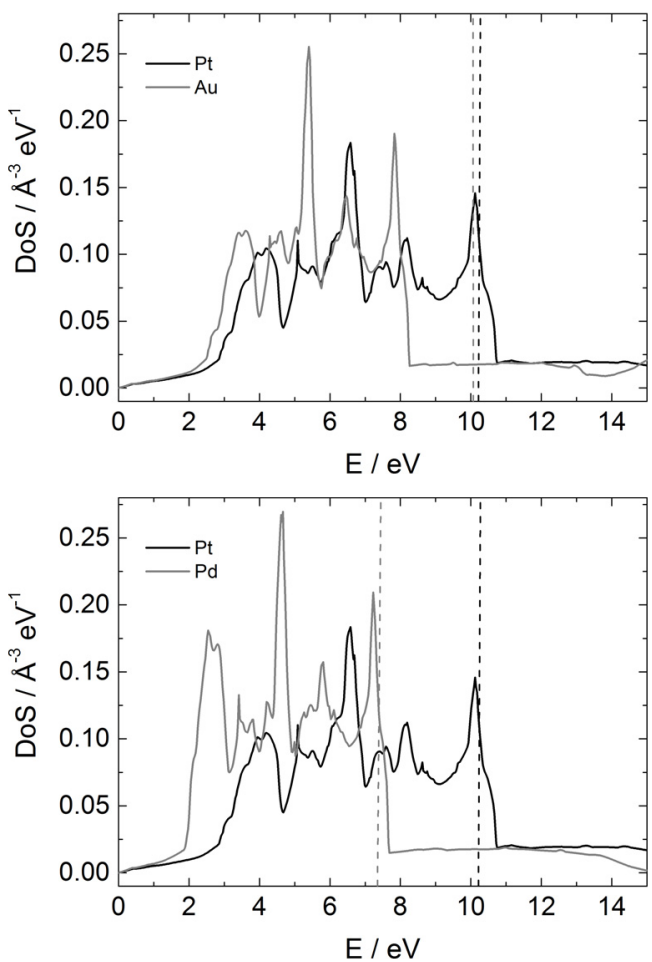

Fig. 1. Calculated density of states grouped by relevance to thermocouple pairs. Top panel: $\mathrm{Au}$ and Pt. Bottom panel: Pt and Pd. Dashed lines show the Fermi energy.

\section{Determination of the electron density of states}

All the calculations of the electron density of states were performed with the ABINIT code [4-6]. The calculations are based on the local density approximation (LDA) [8] combined with the norm-conserving pseudopotential techniques [9]. The calculations were performed for $\mathrm{Pt}, \mathrm{Au}$, and $\mathrm{Pd}$. The valence pseudowave functions are expanded in plane waves up to a cut-off of $400 \mathrm{eV}$ for all elements. A $6 \times 6 \times 6$ Monkhorst-Pack $k$-points mesh is used. The resulting density of states for $\mathrm{Pt}, \mathrm{Au}$, and $\mathrm{Pd}$ are shown in Figure 1. The calculated lattice parameters are $3.916 \AA$, $4.073 \AA$, and $3.864 \AA$, respectively.

\section{Determination of the Fermi energy}

The calculation relies on the fact that the overall number of electrons taking part in conduction is constant. As the temperature increases above absolute zero, this means the value of $E_{F}$ must change in order to ensure that the number of valence electrons is conserved. The value of $E_{F}$ can be determined by balancing the number of electrons able to take part in conduction (determined from the density of states) [7] with the number of electrons in a unit volume calculated from the density as yielded by the lattice parameter calculated by ABINIT:

$$
\int_{-\infty}^{\infty} N(E) P d E=\frac{L \rho N_{A}}{A}
$$



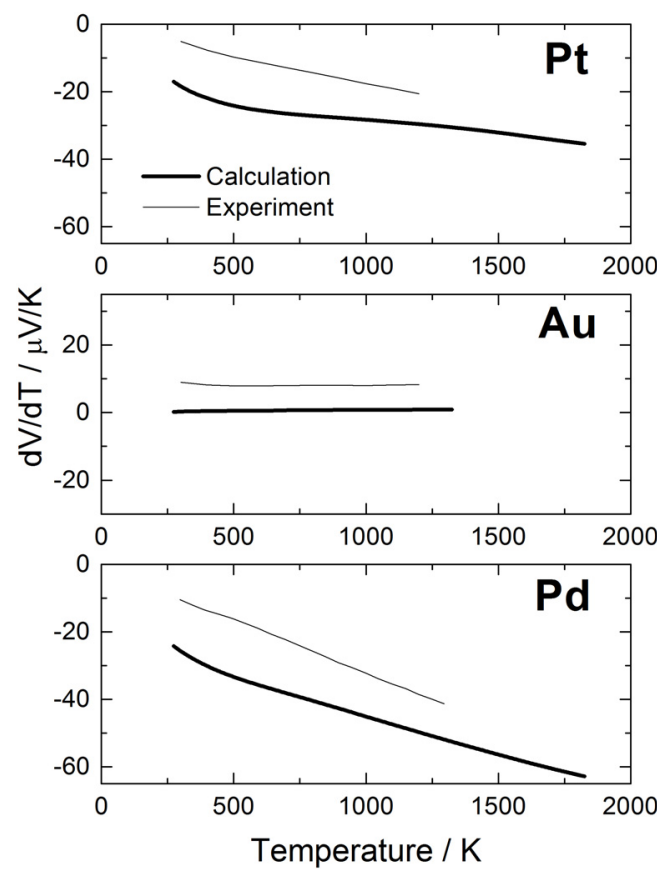

Fig. 2. Absolute thermopower of individual thermoelements $\mathrm{Pt}$ (top), $\mathrm{Au}$ (middle), and Pd (bottom). Thick line shows the thermopower calculated using the method described in the text. The thin grey line shows the experimental values [10-12].

recalling that $N(E)$ denotes the electron density of states, and that $P$ is the (energy dependent) Fermi function of (3). $N_{A}$ is Avogadro's constant, $\rho$ is the zero temperature metal density (determined from the lattice parameter calculated by ABINIT), $A$ is the atomic mass, and there are $L$ valence electrons per atom $(10,11$, and 10 for $\mathrm{Pt}$, $\mathrm{Au}$, and $\mathrm{Pd}$ respectively). The iteration was performed numerically at each temperature. Since the number of conduction electrons must remain constant with temperature according to the principle of electroneutrality, the density is kept constant at its absolute zero temperature value to maintain the number of conduction electrons at the zero temperature value.

\section{Results}

The thermopower (Seebeck coefficient) of each element calculated from expression (8) is shown in Figure 2. The combined thermopower (combined Seebeck coefficient) of the $\mathrm{Au} / \mathrm{Pt}$ and $\mathrm{Pt} / \mathrm{Pd}$ thermocouples is presented in Figure 3. The corresponding relationship between temperature and emf (known as the reference function [11]) is obtained by integrating $d V / d T$ with respect to temperature, and is shown in Figure 4. A reference temperature of $273.15 \mathrm{~K}$ is assumed.

The model gives qualitatively realistic predictions for the temperature dependence of the thermopower for all three metals $\mathrm{Pt}, \mathrm{Au}$, and $\mathrm{Pd}$, and hence both $\mathrm{Au} / \mathrm{Pt}$ and $\mathrm{Pt} / \mathrm{Pd}$ thermocouples, but it is not sufficiently accurate in its current form. The absolute difference between the calculated emf and the reference emf is shown in Figure 5.
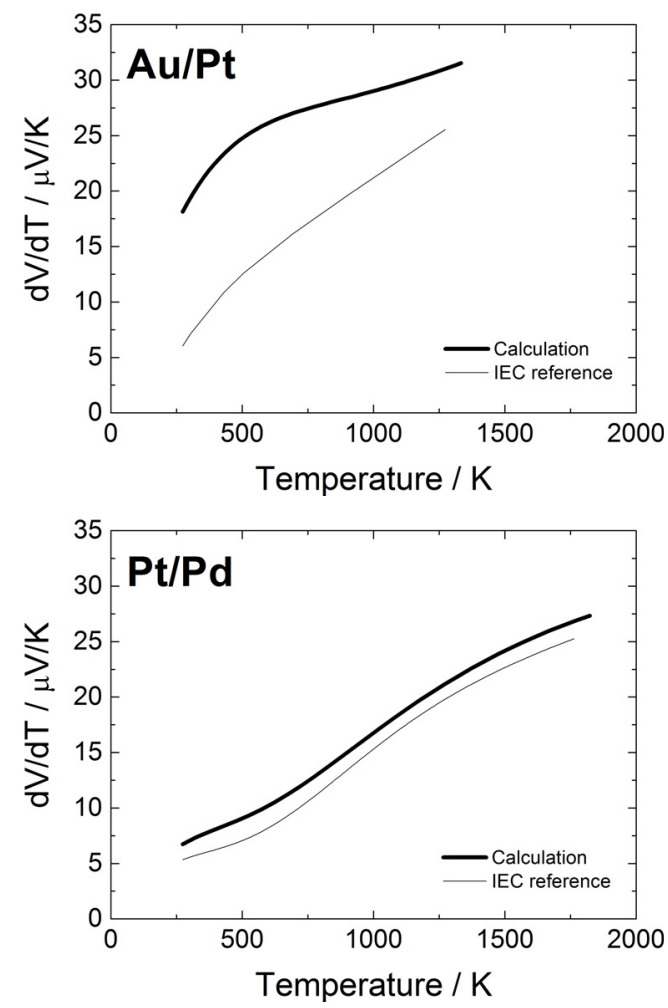

Fig. 3. Temperature dependence of thermopower of $\mathrm{Au} / \mathrm{Pt}$ (top) and $\mathrm{Pt} / \mathrm{Pd}$ (bottom) thermocouples. Thick line shows the thermopower calculated using the method described in the text. Thin grey line shows the experimentally determined values [11].

For the $\mathrm{Au} / \mathrm{Pt}$ thermocouple, the emf difference diverges monotonically as the temperature increases, reaching a maximum corresponding to a temperature difference of about $390{ }^{\circ} \mathrm{C}$. The situation is somewhat better for the $\mathrm{Pt} / \mathrm{Pd}$ thermocouple, with a monotonic divergence reaching a maximum difference of about $130{ }^{\circ} \mathrm{C}$ at a temperature of about $650{ }^{\circ} \mathrm{C}$; the difference remains approximately constant as the temperature increases further. Nonetheless, given the ab initio nature of the calculation and its associated approximations, it may be considered remarkable that the calculated emf even has the correct order of magnitude, and describes the measurements qualitatively.

The fact that the calculation is more accurate for $\mathrm{Pt} / \mathrm{Pd}$ thermocouples probably arises because the calculated thermopower of $\mathrm{Au}$ is the least accurate (this is evident in Fig. 2); there is a similar error introduced in the calculated thermopower of $\mathrm{Pt}$ and $\mathrm{Pd}$, which is largely eliminated when one is subtracted from the other.

\section{Conclusions}

A simple model has been presented which makes a prediction of the thermopower of $\mathrm{Pt}, \mathrm{Au}$, and $\mathrm{Pd}$. The predictions are in good qualitative agreement with the measurements, and describe well certain key features e.g. pronounced changes in the rate of change of thermopower 

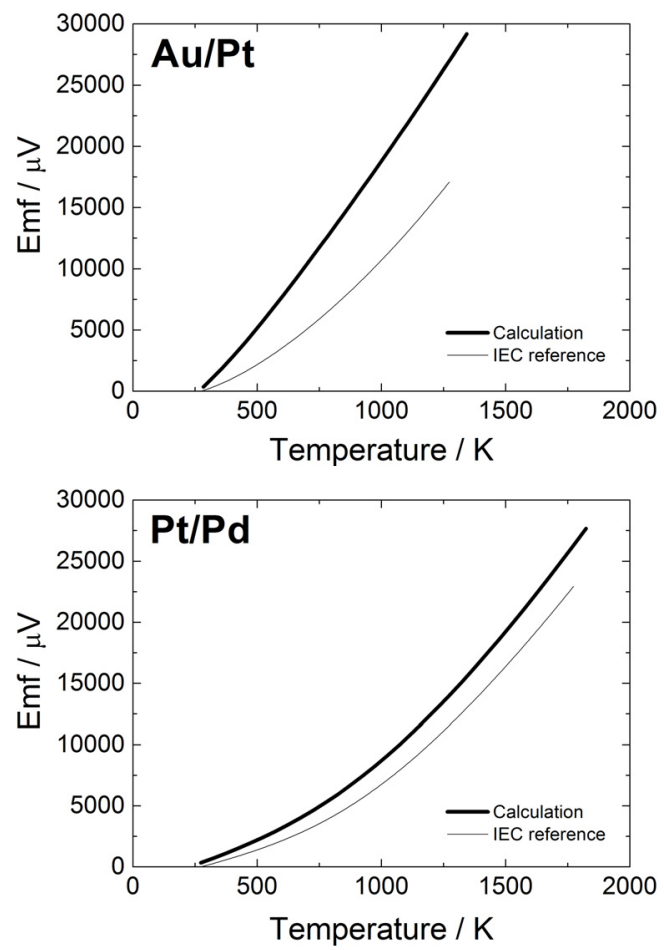

Fig. 4. Temperature dependence of thermocouple emf of $\mathrm{Au} / \mathrm{Pt}$ (top) and Pt/Pd (bottom) thermocouples. Thick line shows the emf calculated using the method described in the text. Thin grey line shows the experimentally determined values [11].

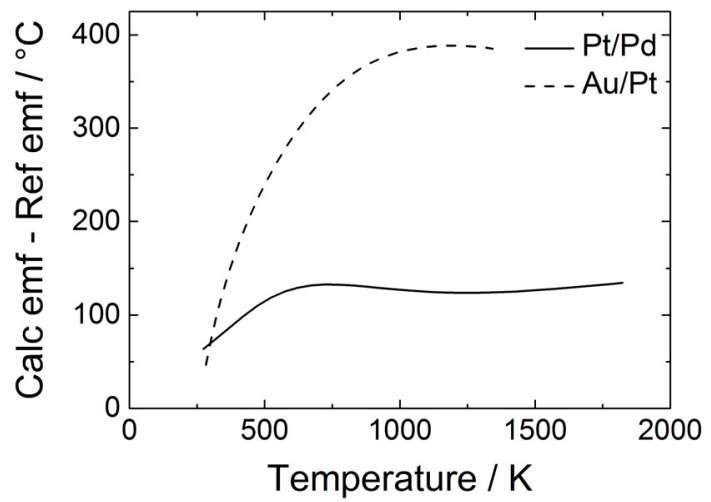

Fig. 5. Difference between the calculated emf and the reference (i.e. measured) emf for the $\mathrm{Au} / \mathrm{Pt}$ thermocouple (dashed line) and $\mathrm{Pt} / \mathrm{Pd}$ thermocouple (solid line).

as a function of temperature, but are not in close enough agreement to be used on a quantitative basis. The current model considers only electron transport, but the thermopower of the metals considered here is also affected by interactions of the charge carriers with the crystal lattice, namely phonon drag $[1,2]$. However, the temperatures of interest for $\mathrm{Au} / \mathrm{Pt}$ and $\mathrm{Pt} / \mathrm{Pd}$ thermocouples $(T>273 \mathrm{~K})$ are well above the Debye temperature of all three metals, so the effect is very small. The assumption that the thermoelements are ideal crystals also limits the applicability of the model.
Nonetheless, it is suggested that the limiting factor in this investigation is the ab initio calculation of the density of states, in which a number of assumptions and approximations are made. The analytical model also contains a number of simplifications. Despite these limitations, the model is sufficiently accurate to provide a qualitative indication of the likely reference function for a given thermocouple. It is noted that another technique based on calculation of the effect of oxide-induced strain on $\mathrm{Pd}$ wire produces much better agreement with the measured thermopower for that metal [13], although it is unclear how that technique could be applied to other metals.

It is hoped that future work will focus on refining the calculation of the density of states, with the aim of providing an accurate, physically based link between thermocouple emf and temperature. A further development opportunity is to extend the calculation to handle alloys as well as pure metals. Provided the resulting material has a crystalline structure, such a calculation is thought to be feasible.

Refined calculations offer the possibility of predicting the relationship between emf and temperature for thermocouples of different compositions, and could have applications for quantitative predictions of calibration drift based on composition change due to harsh environments or ionising radiation. Sufficiently accurate calculations also raise the prospect, in principle, of the thermocouple being used as a primary thermometer, in the sense that the temperature can be related to the emf through fundamental physics, rather than relying on calibrations at fixed temperatures and interpolation by means of ad-hoc polynomial functions.

Acknowledgements. Vlad Sokhan (NPL) is gratefully acknowledged for providing an introduction to the ABINIT code. Valuable discussions with Paul Bramley (Metrosol Limited), Richard Rusby (NPL), and Michael de Podesta (NPL) are acknowledged.

\section{References}

1. D. Pollock, Thermocouples: Theory and Properties (CRC Press, Boca Raton, 1991)

2. J.S. Dugdale, The Electrical Properties of Metals and Alloys (Edward Arnold (Publishers) Limited, London, 1977)

3. R.D. Barnard, Thermoelectricity in Metals and Alloys (Taylor \& Francis Ltd, London, 1972)

4. X. Gonze et al., First-principles computation of materials properties: The ABINIT software project, Comput. Mater. Sci. 25, 478 (2002)

5. X. Gonze et al., ABINIT: First-principles approach to material and nanosystem properties, Comput. Phys. Commun. 180, 2582 (2009)

6. http://www.abinit.org (accessed on 23 March 2010)

7. P. Bramley and S. Clarke, A quantitative model for the thermocouple effect using statistical and quantum mechanics, AIP Conf. Proc. 684, 547 (2003) 
8. D.M. Ceperley, B.J. Alder, Ground state of the electron gas by a stochastic method, Phys. Rev. Lett. 45, 455 (1980)

9. M. Troullier, J.L. Martins, A straightforward method for generating soft transferable pseudopotentials, Solid State Commun. 74, 613 (1990)

10. M.J. Laubitz, Transport properties of pure metals at high temperatures. II. Silver and gold, Can. J. Phys. 47, 2633 (1969)
11. BS EN (IEC) 62460, Temperature-Electromotive Force (EMF) tables for pure-element thermocouple combinations (2008)

12. M.J. Laubitz, T. Matsumara, High-temperature transport properties of palladium, Can. J. Phys. 50, 196 (1972)

13. W.-S. Ohm, K.D. Hill, A Mechanism for the oxidationrelated influence on the thermoelectric behavior of palladium, Int. J. Thermophys. 31, 1402 (2010) 